\title{
Micro-interface temperature field of catalytic particle under self- rotation regulation
}

\author{
Lei $\mathrm{Xu}^{1}$, Hualin $\mathrm{Wang}^{2}$, and Yuan Huang, \\ ${ }^{1}$ School of Mechanical and Power Engineering, East China University of Science and Technology, Shanghai 200237, China \\ ${ }^{2}$ School of Resources and Environmental Engineering, East China University of Science and Technology, Shanghai 200237, China \\ ${ }^{3}$ School of Chemistry and Molecular Engineering, East China University of Science and Technology, Shanghai 200237, China
}

\begin{abstract}
The micro-interface formed inside and on the surface of the catalytic particle is the place where the catalytic reaction proceeds. The micro-interface temperature is one of the important factors determining the reaction efficiency. Numerical simulation was used to investigate the fluid-solid coupled heat transfer law of micro-interface under the regulation of spherical particles' self-rotation. The results show it takes up to $4.78 \mathrm{~s}$ that the average surface temperature of non-rotating particle with a diameter of $3 \mathrm{~mm}$ decreases from $300{ }^{\circ} \mathrm{C}$ to $150{ }^{\circ} \mathrm{C}$, which is the lowest temperature required for high-efficiency selective catalytic reduction reaction of $\mathrm{NO}_{x}$ with $\mathrm{NH}_{3}$, while self-rotation can reduce the cooling rate of particles. When the direction of particle self-rotation is perpendicular to gas velocity, as the self-rotation speed increases, the high-temperature area of the particle surface diffuses more fully to the latitude direction. Significant effect can be made as the self-rotation speed reaches $5 \mathrm{rad} / \mathrm{s}$, the uniformity of the surface temperature can be increased by $27.1 \% \sim 37.7 \%$ compared with non-rotating particle, and the uniformity can be increased by a maximum of $49.5 \%$ at a self-rotation speed of $500 \mathrm{rad} / \mathrm{s}$.
\end{abstract}

\section{Introduction}

The flue gas temperature of non-power industry is low $\left(120 \sim 250{ }^{\circ} \mathrm{C}\right)$, and it can be lowed than $100{ }^{\circ} \mathrm{C}$ in the $\mathrm{deNO}_{\mathrm{x}}$ process of terminal arrangement [1]. At present, low-temperature activity of commercial deNO $\mathrm{x}_{\mathrm{x}}$ catalysts have poor stability. $\mathrm{NH}_{3}-\mathrm{SCR}$ deNO x process of lowtemperature flue gas has problems such as high overall energy consumption for heating the flue gas and poor $\mathrm{H}_{2} \mathrm{O}$ and $\mathrm{SO}_{2}$ resistance of the catalyst at low temperatures [2-5]. The mainstream of research is still to develop catalysts with low active temperature and strong anti-toxic performance, the lower limit of temperature for deNO ${ }_{x}$ efficiency reaching $90 \%$ has been reduced to $150 \sim 180{ }^{\circ} \mathrm{C}$ [6-8]. Particle self-rotation in cyclones can enhance the mass transfer process of adsorption $[9,10]$ and interface update $[11,12]$. This work changes the idea of high energy consumption for heating flue gas, based on the high-speed self-rotation of particles in the swirling field $[13,14]$ and SCR reaction mainly proceeding on the micro-interface of catalysts [15], a cyclonic fluidized reactor and process using catalytic particles as the circulating heat carrier for deNO $\mathrm{N}_{\mathrm{x}}$ of lowtemperature flue gas are proposed.

High-temperature catalytic particles directly contact low-temperature flue gas in the reactor, so the particles are in a rapid cooling process. In order to fully analyze the flow field and temperature field near the particle surface and accurately simulate particle motion and heat transfer, Direct Numerical Simulation was developed $[16,17]$. Shao et al. [18] proposed finite size particle method and double distribution function coupling model of lattice Boltzmann method, obtained the heat diffusion process around an isothermal hot sphere submerged in a cold fluid to confirm the thermal boundary condition between particle and fluid two phases. Thiam et al. [19] used a Lagrangian VOF approach for simulating gassolid heat exchanges in dense regimes, fully resolving fluid velocity and temperature evolving within random arrays of fixed particles. Tavassoli et al. [20] simulated the surface heat transfer coefficient of 54 spherical fixed particles randomly arranged in a square cavity by using the embedded boundary method based on multiple direct forces and multiple heat sources.

Although the above studies have conducted deep explorations on the heat transfer process of gas-solid two-phase flow, the research on the effect of particle self-rotation on fluid-solid coupled heat transfer is still scarce. In this work, CFD is used to establish a fluidsolid coupled heat transfer model under the regulation of particles' self-rotation speed and direction. The effect of particle self-rotation on the micro-interface temperature field of multiple catalytic particles regularly arranged is studied.

\footnotetext{
* Corresponding author: yuanhuang@ecust.edu.cn
} 
(a)

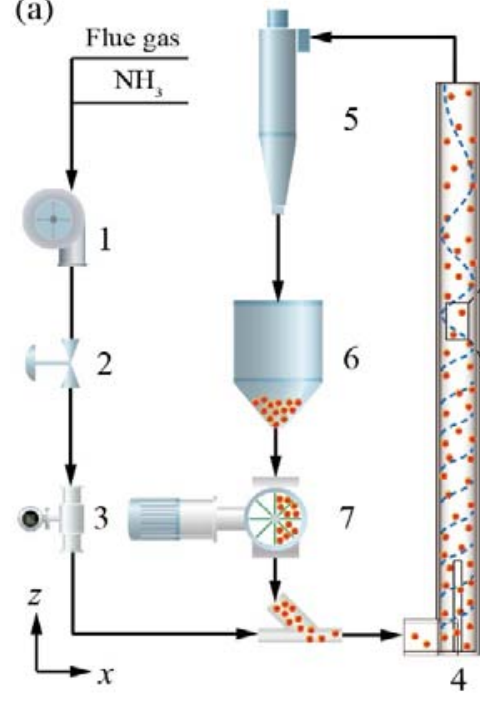

(b)

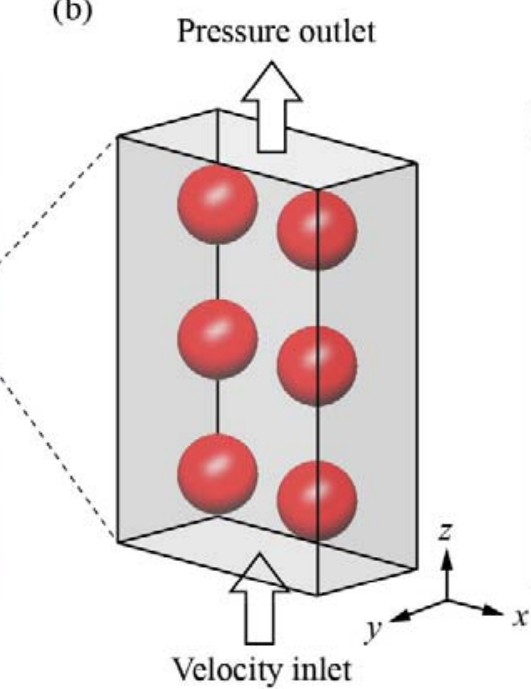

Velocity inlet

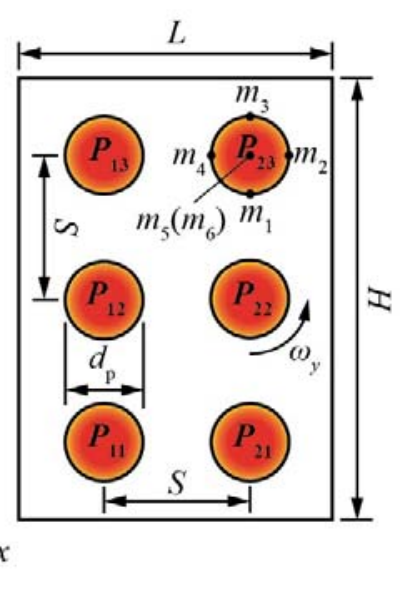

Fig. 1. Gas-solid coupled heat transfer: (a) process flow diagram; (b) 3D structure diagram of simulated space.

1 - blower; 2 - valve; 3 - flow meter; 4 - cyclonic fluidized reactor; 5 - cyclone separator; 6 - heat storage tank; 7-feed valve

\section{Numerical method}

$\mathrm{DeNO}_{\mathrm{x}}$ process of low-temperature flue gas with thermal catalyst circulating in cyclonic fluidized reactor is shown in Fig. 1a. The low-temperature flue gas and $\mathrm{NH}_{3}$ are mixed at a ratio of 1: 1 , the catalyst particles are heated to $300{ }^{\circ} \mathrm{C}$ in the heating storage tank 6 , and continuously fed into the mixed gas stream by the feed valve $7 . \mathrm{NO}_{\mathrm{x}}$ in the flue gas and $\mathrm{NH}_{3}$ complete the SCR reaction on the micro-interface of catalyst particles. The local space of the cyclonic fluidized reactor 4 as the research object of gas-solid coupled heat transfer is shown in Fig. 1b. The following assumptions are made in the numerical simulation: (1) the catalyst particles only rotate but not migrate; (2) the surface of the catalyst particles is smooth; (3) the arrangement of the particles is regular.

\subsection{Model description}

In the geometric model shown in Fig. 1b, the diameter of the sphere is $d_{\mathrm{p}}$, the distance between two spheres in the $y$-axis and $z$-axis direction is $S$, the initial temperature of the sphere is $T_{\mathrm{p}}$, the inlet velocity and temperature of air are $u$ and $T$ respectively. The six spheres are numbered $P_{11}, P_{21}, P_{12} \ldots P_{23}$. Six temperature monitoring points $m_{1}$, $m_{2}, m_{3} \ldots m_{6}$ are set on the surface of each sphere. The length $L$ of the model is equal to $2 S$, the width $W$ is equal to $S$, and the height $H$ is equal to $3 S$. The model has the following relationship:

$$
\frac{\pi d_{\mathrm{p}}^{3}}{6 S^{3}}=1-\varepsilon
$$

where $\varepsilon$ is bed voidage of fluidized bed, in the calculation of fluidization parameters, according to Pranovsky's general formula:

$$
R e=\frac{\rho u d_{\mathrm{p}}}{\mu}=\frac{\left(367+k_{3} A r \varepsilon^{4.75}\right)^{1 / 2}-19.15}{0.588 k_{3}}
$$

$$
A r=\frac{\rho\left(\rho_{\mathrm{p}}-\rho\right) g d_{\mathrm{p}}{ }^{3}}{\mu^{2}}
$$

where $R e$ is the Reynolds number, $k_{3}$ is the shape correction coefficient of particle, $u$ is the operating gas velocity, $A r$ is the Archimedes constant, $d_{\mathrm{p}}$ is the average diameter of particles; $\rho_{\mathrm{p}}$ is the density of particles, $\rho$ is the gas density, and $\mu$ is the aerodynamic viscosity of gas.

According to physical parameters of $\mathrm{deNO}_{\mathrm{x}}$ catalyst and air at $80{ }^{\circ} \mathrm{C}$, when $d_{\mathrm{p}}$ is equal to $3 \mathrm{~mm}$ and $\varepsilon$ is 0.9 , from the calculation of equations (1) to (3), it can be obtained that $S$ is equal to $5.209 \mathrm{~mm}$, and $u$ is equal to $7.88 \mathrm{~m} / \mathrm{s}$.

\subsection{Governing equations and boundary conditions}

Since the simulation involves two phases, the grid is divided into two types of regions. In order to allow energy transfer between the two regions, coupling is performed through the interface couple-wall method in Fluent. The fluid medium is atmospheric air at $80^{\circ} \mathrm{C}$, the sphere material is deNO ${ }_{x}$ carbon-based catalyst with a density of $2204 \mathrm{~kg} / \mathrm{m}^{3}$. The numerical model couples the continuity equation, momentum equation, and energy equation, as follows:

$$
\begin{gathered}
\frac{\partial\left(\rho u_{j}\right)}{\partial x_{i}}=0 \\
\frac{\partial\left(\rho u_{i} u_{j}\right)}{\partial x_{j}}=-\frac{\partial p}{\partial x_{j}}+\frac{\partial}{\partial x_{j}}\left[\left(\mu+\mu_{t}\right) \frac{\partial u_{i}}{\partial x_{j}}\right] \\
\frac{\partial\left(\rho u_{j} T\right)}{\partial x_{j}}=\frac{\partial}{\partial x_{j}}\left[\left(\frac{\mu}{P r}+\frac{\mu_{t}}{P r_{t}}\right) \frac{\partial C_{p} T}{\partial x_{j}}\right]
\end{gathered}
$$

The turbulence equation uses a standard $k-\varepsilon$ model:

$$
\frac{\partial(\rho k)}{\partial t}+\frac{\partial\left(\rho k u_{i}\right)}{\partial x_{i}}=\frac{\partial}{\partial x_{j}}\left[\left(\mu+\frac{\mu_{t}}{\sigma_{k}}\right) \frac{\partial k}{\partial x_{j}}\right]+G_{k}-\rho \xi
$$




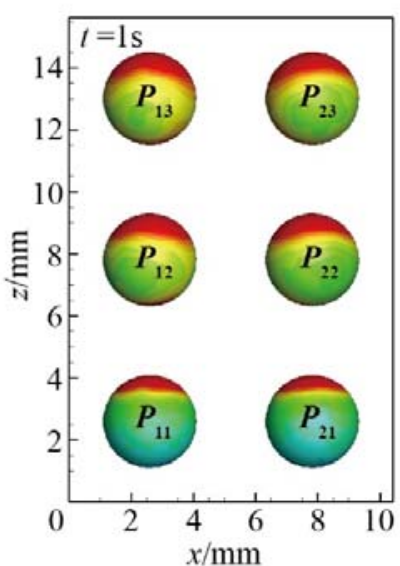

(a) $\omega=0 \mathrm{rad} / \mathrm{s}$

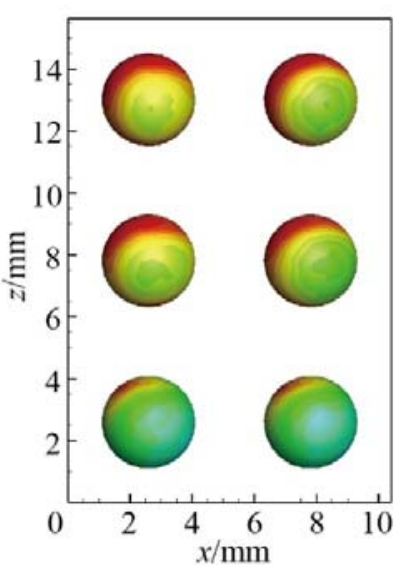

(b) $\omega_{y}=3 \mathrm{rad} / \mathrm{s}$

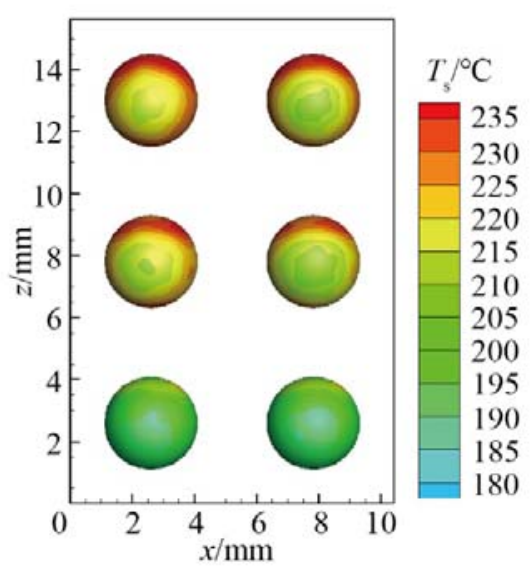

(c) $\omega_{y}=100 \mathrm{rad} / \mathrm{s}$

Fig. 2. Surface temperature nephograms of particles at different self-rotation speeds.

$$
\frac{\partial\left(\rho \xi u_{i}\right)}{\partial t}=\frac{\partial}{\partial x_{j}}\left[\left(\mu+\frac{\mu_{t}}{\sigma_{\xi}}\right) \frac{\partial \xi}{\partial x_{j}}\right]+\frac{\xi}{k}\left(C_{1 \xi} G_{k}-C_{2 \xi} \xi \rho\right)
$$

where $t$ is time, $p$ is pressure, $k$ is turbulent pulsating kinetic energy; $\xi$ is dissipation rate of turbulent pulsating kinetic energy, $C_{p}$ is pressure coefficient, $G_{k}$ is turbulent kinetic energy generated by average velocity gradient, the subscripts $i$ and $j$ can be taken as 1,2, and 3, which represent the $x$-axis, $y$-axis, and $z$-axis direction respectively, $\mu_{t}$ is the turbulent viscosity, which is defined as:

$$
\mu_{t}=\rho C_{\mu} \frac{k^{2}}{\xi}
$$

the model parameters $C_{1 \xi}, C_{2 \xi}, C_{\mu}, \sigma_{k}$, and $\sigma_{\xi}$ generally are taken as the default values: $1.44,1.92,0.09$, 1.0 , and 1.3 , respectively.

The solution process is transient. SIMPLE algorithm is used as the calculation method to deal with the coupling of pressure and velocity. Second Order Upwind format is used in the diffusion and convection terms when discreting the diffusion equation. In addition, the inlet is set as velocity inlet, and $u$ is set to $7.88 \mathrm{~m} / \mathrm{s}$, the outlet is set as pressure outlet. $T_{\mathrm{p}}$ and $T$ are set to $300{ }^{\circ} \mathrm{C}$ and $80{ }^{\circ} \mathrm{C}$ respectively, the wall surface other than the sphere is set to be adiabatic, and no slip wall boundary condition is used on the wall surface. In order to capture the temperature change of the sphere surface, a transient calculation was made. To ensure that the Courant number is less than 2.5 , the time step $\Delta t$ of the transient calculation is taken as $0.0002 \mathrm{~s}$, the convergence criterion of the velocity term is less than $10^{-4}$, and the convergence criterion of energy residual, turbulent kinetic energy, and dissipation rate are less than $10^{-6}$.

\section{Results and discussions}

\subsection{Effect of self-rotation speed on particle surface temperature field}

The comparison of the particle surface temperature nephograms at a self-rotation speed $\omega$ of 0,3 , and 100 $\mathrm{rad} / \mathrm{s}$ is shown in Fig. 2. It can be seen that the difference between two particles is little in the $x$-axis direction of single model, while the three particles in the $z$-axis direction show a slower temperature decrease as the distance from the inlet is longer. When particles don't rotate, the surface temperature of particles' upper part decreases more slowly, the temperature span of single particle is large. When $\omega_{y}$ is equal to $3 \mathrm{rad} / \mathrm{s}$, it is obvious that the high-temperature region has a tendency to diffuse toward the latitude direction, the uniformity of the particle surface temperature is improved. And when the self-rotation speed $\omega_{y}$ is increased to $100 \mathrm{rad} / \mathrm{s}$, the high temperature region is mainly distributed on the equator, the closer the surface is to the self-rotation axis, the lower the surface temperature is, and the uniformity of the surface temperature is integrally better.

The data of temperature monitoring points $m_{1}, m_{2}$, $m_{3} \ldots m_{6}$ on the surface of spheres $P_{21}, P_{22}, P_{23}$ was analyzed and integrated, $\bar{T}_{\mathrm{s}}$ and $\sigma$ are defined as the the average temperature and temperature standard deviation of single particle's surface, respectively. $\Delta \bar{T}_{\mathrm{s}}$ is defined as the difference between $\bar{T}_{\mathrm{s}}$ at different self-rotation speeds and $\bar{T}_{\mathrm{s}}$ of non-rotating particle.

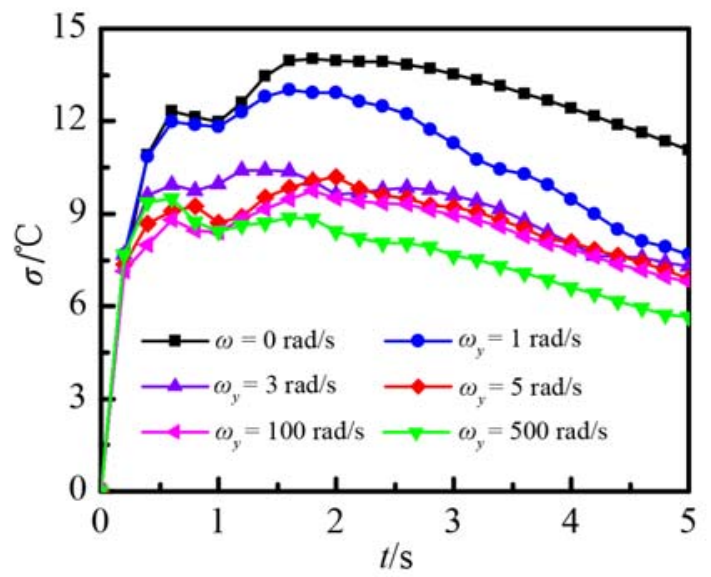

Fig. 3. Surface temperature standard deviation of sphere $P_{23}$ at different self-rotation speeds. 


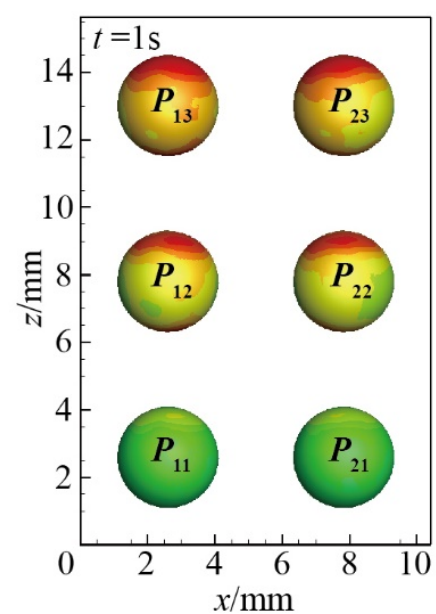

(a) $\omega_{x}=100 \mathrm{rad} / \mathrm{s}$

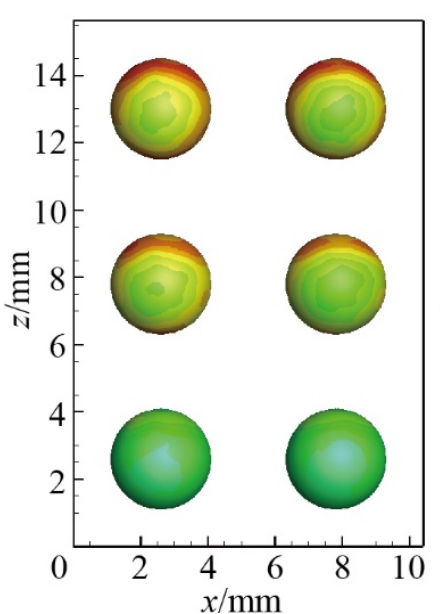

(b) $\omega_{y}=100 \mathrm{rad} / \mathrm{s}$

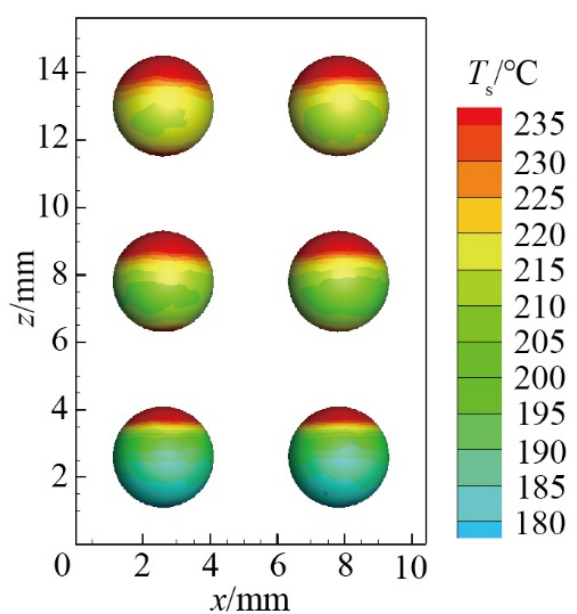

(c) $\omega_{z}=100 \mathrm{rad} / \mathrm{s}$

Fig. 4. Surface temperature nephograms of particles in different self-rotation directions.

Comparison of $\sigma$ of sphere $P_{23}$ at different selfrotation speeds is shown in Fig. 3. $\sigma$ rises sharply with time, and then decreases slowly after a relatively smooth transition in the range of $0.4 \mathrm{~s}$ to $1.6 \mathrm{~s}$. With the increase of the self-rotation speed, the uniformity of particles' surface temperature gradually increases, but the uniformity increases slowly after $\omega_{y}$ is greater than 5 $\mathrm{rad} / \mathrm{s}$. That is, the increase of the self-rotation speed can significantly improve the uniformity, however there is a limit to the improvement. Significant effect can be made as the self-rotation speed reaches $5 \mathrm{rad} / \mathrm{s}$, the uniformity of the surface temperature can be increased by $27.1 \%$ $37.7 \%$ compared with non-rotating particle after $1 \mathrm{~s}$, and the uniformity can be increased by a maximum of $49.5 \%$ at a self-rotation speed of $500 \mathrm{rad} / \mathrm{s}$. The contact area during the gas-solid phase catalytic reaction is crucial to improve the reaction efficiency. The improvement of the uniformity of particles' surface temperature will undoubtedly have a positive effect on the utilization of the micro-interface of the catalyst particles during the cooling process.

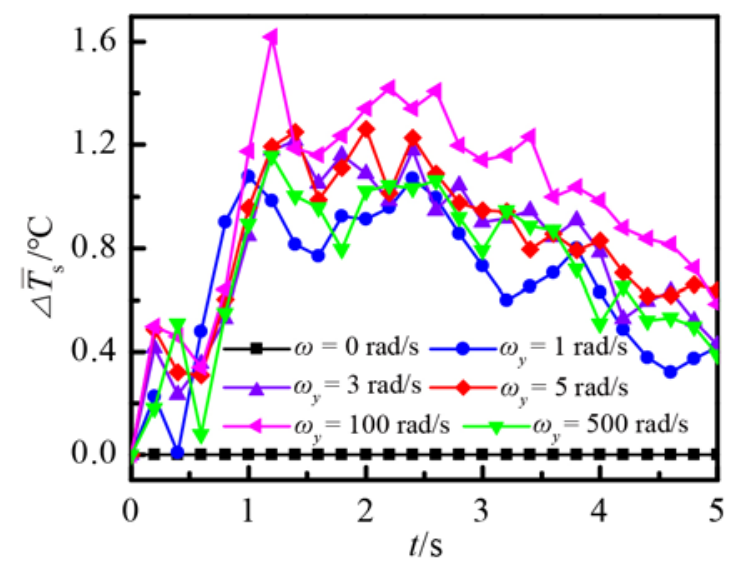

Fig. 5. Average surface temperature of sphere $P_{23}$ at different self-rotation speeds.

Comparison of $\Delta \bar{T}_{\mathrm{s}}$ of sphere $P_{23}$ at different selfrotation speeds is shown in Fig. 5. $\Delta \bar{T}_{\mathrm{s}}$ shows a small fluctuation, first rises rapidly and then slowly decreases, that is, the average surface temperature of particles decreases more slowly when particles have rotational motion. For instance, the temperature difference between particle at a self-rotation speed of $100 \mathrm{rad} / \mathrm{s}$ and nonrotating particle can reach up to $1.62{ }^{\circ} \mathrm{C}$ at $1.2 \mathrm{~s}$. As $\omega_{y}$ increases below $100 \mathrm{rad} / \mathrm{s}, \Delta \bar{T}_{\mathrm{s}}$ generally shows a gradually increasing trend.

\subsection{Effect of self-rotation direction on particle surface temperature field}

The comparison of the particle surface temperature nephograms in the directions of $x, y$, and $z$-axis is shown in Fig. 4. When particles' self-rotation axis is the $z$-axis direction, the same rules as non-rotating particles are displayed, but the surface temperature distribution in the latitude direction is relatively more uniform due to selfrotation. When particles' self-rotation axis is the $x$-axis or $y$-axis direction, high-temperature region diffuses fully toward the latitude direction, the uniformity of the particle surface temperature is much improved compared to the particle rotating around the $z$-axis direction. It shows that the self-rotation direction parallel to the gas velocity is not beneficial to the uniformity of surface temperature.

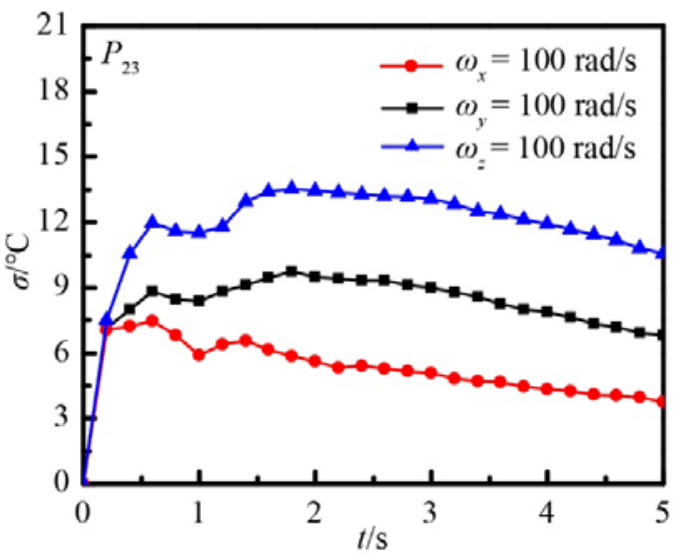

Fig. 6. Surface temperature standard deviation of sphere $P_{23}$ in different self-rotation directions. 
Comparison of $\sigma$ of sphere $P_{23}$ in different selfrotation directions is shown in Fig. 6. Surface temperature uniformity of particle rotating around $x$-axis direction is best, followed by the $y$-axis direction. The symmetry of the sphere distribution in the $y$-axis direction isn't considered in the geometric model, resulting in a slight difference between the surface temperature field of particles rotating around $x$-axis and $y$-axis directions. The surface temperature characteristics of particle rotating around the $z$-axis direction are same as non-rotating particle, uniformity of the surface temperature is very poor, and Fig. 7 shows the temperature drop comparison of them, the difference is still very small. It takes up to $4.78 \mathrm{~s}$ that the average surface temperature decreases from $300{ }^{\circ} \mathrm{C}$ to $150{ }^{\circ} \mathrm{C}$, which is the lowest temperature required for highefficiency SCR reaction of $\mathrm{NO}_{x}$ with $\mathrm{NH}_{3}$. So particle self-rotation has a marginal effect on the surface average temperature drop.

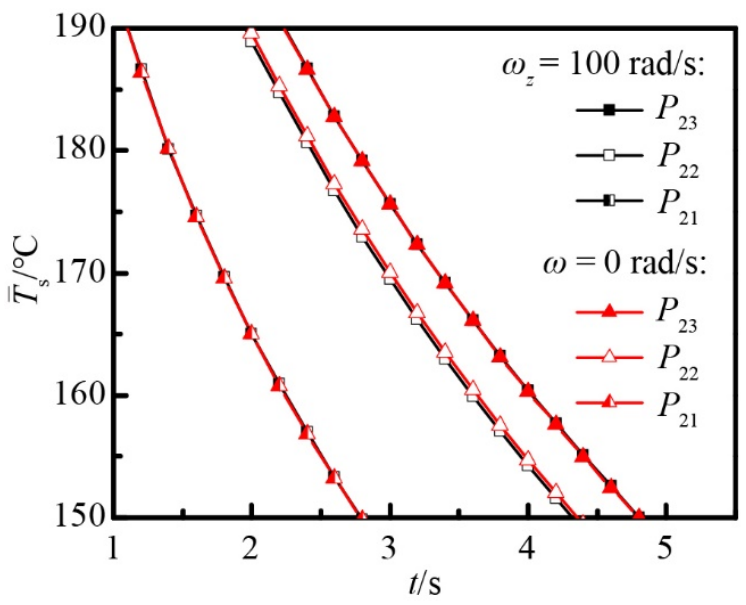

Fig. 7. Temperature drop characteristics of particle rotating around $z$-axis direction and non-rotating particle

\section{Conclusions}

In this study, a numerical simulation method was developed to investigate the effects of particle selfrotation speed and direction on particles' surface temperature field. When the direction of particle selfrotation is perpendicular to the direction of gas velocity, with the increase of the self-rotation speed, temperature uniformity of catalyst surface gradually get improved. Significant effect can be made as the self-rotation speed reaches $5 \mathrm{rad} / \mathrm{s}$, the temperature uniformity can be increased by $27.1 \% \sim 37.7 \%$ compared with non-rotating particle after $1 \mathrm{~s}$, and the uniformity can be increased by a maximum of $49.5 \%$ at a self-rotation speed of 500 $\mathrm{rad} / \mathrm{s}$. When the direction of particle self-rotation is parallel to gas velocity, temperature characteristics of catalyst surface are basically the same as those of the non-rotating particle. By regulating the self-rotation of high-temperature catalyst, micro-interface of catalyst can uniformly transfer heat, ensuring that the catalyst microinterface temperature is in the active temperature range. The results have guiding significance for the feasibility and regulation of low-temperature flue gas deNO method with thermal catalyst circulating in the cyclonic fluidized reactor.

\section{Acknowledgment}

This work has been supported by the Shanghai Sailing Program (Grant Nos. 19YF1412000).

\section{References}

1. R.M. Heck, Catal. Today 53(4), 519-523 (1999)

2. J. Klinik, B. Samojeden, T. Grzybek, et al., Catal. Today 176(1), 303-308 (2011)

3. Z. Huang, Z. Liu, X. Zhang, et al., Appl. Catal. BEnviron. 63(3-4), 260-265 (2006)

4. Z. Zhu, H. Niu, Z. Liu, et al., J. Catal. 195(2), 268278 (2000)

5. P. Li, Q. Liu, Z. Liu, Chem. Eng. J. 181-182(1), 169-173 (2012)

6. X. Tang, J. Hao, H. Yi, et al., Catal. Today 126(3-4), 406-411 (2007)

7. L. Zhu, B. Huang, W. Wang, et al., Catal. Commun. 12(6), 394-398 (2011)

8. X. Gao, S. Liu, Y. Zhang, et al., Catal. Today 175(1), 164-170 (2011)

9. P. Qian, L. Ma, Y. Liu, et al., Chem. Eng. Technol. 39(5), 953-959 (2016)

10. L. Ma, M.Y. He, P.B. Fu, et al., Sep. Purif. Technol. , 235 (2020)

11. D. Shi, Y. Huang, H.L. Wang, et al., Sep. Purif. Technol. 210, 117-124 (2019)

12. P.B. Fu, H.L. Wang, J.P. Li, et al., J. Clean. Prod. 190, 689-702 (2018)

13. S. Parsa, E. Calzavarini, F. Toschi, et al., Phys. Rev. Lett 109, 1-5 (2012)

14. C.R. Meyer, M.L. Byron, E.A. Variano, Limnol. Oceanogr 3, 89-102 (2013)

15. J. Yang, H. Ma, Y. Yamamoto, et al., Chem. Eng. J. 230, 513-521 (2013)

16. P. Bagchi, M.Y. Ha, S. Balachandar, J. Fluids Engineering 123, 347-358 (2001)

17. S. Balachandar, M.Y. Ha, Phys. Fluids 13(12), 3714-3728 (2001)

18. B.L. Shao, S.Y. Wang, R.C. Tian, et al., Appl. Therm. Eng. , 157 (2019)

19. E.I. Thiam, E. Masi, E. Climent, et al., Acta Mech. 230, 541-567 (2019)

20. H. Tavassoli, S.H.L. Kriebitzsch, M.A. Van Der Hoef, et al., Int. J. Multiphas. Flow 57, 29-37 (2013) 